\title{
A note on annihilators in distributive nearlattices
}

\author{
Ismael Calomino and Sergio Celani
}




\title{
A NOTE ON ANNIHILATORS IN DISTRIBUTIVE NEARLATTICES
}

\author{
ISMAEL CALOMINO AND SERGIO CELANI
}

Received 10 September, 2014

\begin{abstract}
In this note we propose a definition of relative annihilator in distributive nearlattices with greatest element different from that given in [6] and we present some new characterizations of the distributivity. Later, we study the class of normal and p-linear nearlattices, the lattice of filters and semi-homomorphisms that preserve annihilators.
\end{abstract}

2010 Mathematics Subject Classification: 06A12; 03G10; 06D50

Keywords: distributive nearlattice, relative annihilator, prime ideal, semi-homomorphism

\section{INTRODUCTION AND PRELIMINARIES}

Abbott in [1] established a correspondence between the class of Tarski algebras, or implication algebras, and join-semilattices in which every principal filter is a Boolean lattice with respect to the induced order. There is an algebraic structure that generalizes the class of Tarski algebras: nearlattices. A nearlattice is a join-semilattice in which every principal filter is a lattice. The class of nearlattices forms a variety that has been studied in [9] and [11] by Cornish and Hickman, and in [4], [6] and [7] by Chajda, Kolařík, Halaš and Kühr. In [2] the authors showed that the axiom systems given in [11] and [4] are dependent and that the variety of nearlattices is 2-based. An important class of nearlattices is the class of distributive nearlattices. Recently in [3], a full duality between distributive nearlattices with greatest element and certain topological spaces with a distinguished basis was developed.

It is well known that the notion of distributivity in a lattice can be characterized in different ways, for example, a lattice $A$ is distributive if and only if the lattice $\operatorname{Fi}(A)$ of all filters of $A$ is distributive. Another way is through some special subsets, called annihilators. In a lattice $A$, the annihilator of a relative to $b$ is defined as the set $\langle a, b\rangle=\{x \in A: x \wedge a \leq b\}$. In [12], Mandelker studied the properties of relative annihilators and characterized the distributivity of a lattice in terms of its relative annihilators. To be more precise, a lattice $A$ is distributive if and only if $\langle a, b\rangle$ is an ideal of $A$ for all $a, b \in A$. These results were generalized by Varlet to the

Both authors were partially supported by PIP 112-201101-00636 CONICET. 
class of distributive semilattices ([14]) and subsequently to the variety of distributive nearlattices by Chajda and Kolař́ík ([6]).

The main aim of this paper is to propose a definition of relative annihilator in distributive nearlattices with greatest element different from that given in [6]. In Section 2 we introduce the relative annihilators and we develop new characterizations. In Section 3 we present the class of normal and p-linear nearlattices. In Section 4 we study the lattice of filters of a distributive nearlattice. Finally, in Section 5 we characterize the semi-homomorphisms that preserve annihilators.

Given a poset $\langle X, \leq\rangle$, a set $Y \subseteq X$ is called increasing if it is closed under $\leq$, i.e., if for every $x \in Y$ and every $y \in X$, if $x \leq y$ then $y \in Y$. Dually, $Y \subseteq X$ is said to be decreasing if for every $x \in Y$ and every $y \in X$, if $y \leq x$ then $y \in Y$. The complement of a subset $Y \subseteq X$ will be denoted by $X-Y$. For each $Y \subseteq X$, the increasing (decreasing) set generated by $Y$ is $[Y)=\{x \in X: \exists y \in Y(y \leq x)\}$ $((Y]=\{x \in X: \exists y \in Y(x \leq y)\})$. If $Y=\{y\}$, then we will write $[y)$ and $(y]$ instead of $[\{y\})$ and $(\{y\}]$, respectively.

A join-semilattice with greatest element is an algebra $\langle A, \vee, 1\rangle$ of type $(2,0)$ such that the operation $\vee$ is idempotent, commutative, associative and $x \vee 1=1$ for all $x \in A$. The binary relation $\leq$ defined by $x \leq y$ if and only if $x \vee y=y$ is a partial order. In what follows, we shall write simply semilattice.

A filter of a semilattice $A$ is a subset $F \subseteq A$ such that $1 \in F, F$ is increasing and if $x, y \in F$ then $x \wedge y \in F$, whenever $x \wedge y$ exists. The set of all filters of $A$ is denoted by $\operatorname{Fi}(A)$. Let $X$ be a non-empty subset of $A$. The least filter containing $X$ is called the filter generated by $X$ and will be denoted by $F(X)$. Note that if $X=\{a\}$ then $F(\{a\})=[a)$, called the principal filter of $a$.

A subset $I$ of $A$ is called an ideal if $I$ is decreasing and if $x, y \in I$ then $x \vee y \in I$. The least ideal containing $X$ is called the ideal generated by $X$ and will be denoted by $I(X)$. We shall say that a non-empty proper ideal $P$ is prime if for all $x, y \in A$, if $x \wedge y \in P$, whenever $x \wedge y$ exists, then $x \in P$ or $y \in P$. We will denote by $\operatorname{Id}(A)$ and $X(A)$ the set of all ideals and prime ideals of $A$, respectively. Finally, we will say that a non-empty ideal $I$ of $A$ is maximal if it is proper and for every $J \in \operatorname{Id}(A)$, if $I \subseteq J$ then $J=I$ or $J=A$. We denote by $\operatorname{Idm}(A)$ the set of all maximal ideals of $A$. It is easy to prove that every maximal ideal is prime.

Definition 1. A nearlattice is a semilattice $A$ such that for each $a \in A$ the principal filter $[a)=\{x \in A: a \leq x\}$ is a bounded lattice.

The class of nearlattices forms a variety since every nearlattice $A$ can be described as an algebra with one ternary operation: if $x, y, a \in A$, the element $m(x, y, a)=$ $(x \vee a) \wedge_{a}(y \vee a)$ is correctly defined because $x \vee a, y \vee a \in[a)$ and $[a)$ is a lattice, where $\wedge_{a}$ denotes the meet in $[a)$.

Proposition 1 ([2]). Let A be a nearlattice. The following identities are satisfied:

(1) $m(x, y, x)=x$, 
(2) $m(m(x, y, z), m(y, m(u, x, z), z), w)=m(w, w, m(y, m(x, u, z), z))$,

(3) $m(x, x, 1)=1$.

Conversely, let $\langle A, m, 1\rangle$ be an algebra of type $(3,0)$ satisfying the identities (1)(3). If we define $x \vee y=m(x, x, y)$, then $A$ is a semilattice and for each $a \in A,[a)$ is a bounded lattice, where the meet of $x, y \in[a)$ is $x \wedge a y=m(x, y, a)$. Hence $A$ is a nearlattice.

Definition 2. Let $A$ be a nearlattice. We call $A$ distributive if the principal filter $[a)=\{x \in A: a \leq x\}$ is a bounded distributive lattice for each $a \in A$.

Theorem 1 ([7]). Let $A$ be a nearlattice. Then $A$ is distributive if and only if satisfies either of the following identities:

(1) $m(x, m(y, y, z), w)=m(m(x, y, w), m(x, y, w), m(x, z, w))$,

(2) $m(x, x, m(y, z, w))=m(m(x, x, y), m(x, x, z), w)$.

Theorem 2 ([10]). Let $A$ be a distributive nearlattice. Let $I \in \operatorname{Id}(A)$ and let $F \in \operatorname{Fi}(A)$ such that $I \cap F=\varnothing$. Then there exists $P \in X(A)$ such that $I \subseteq P$ and $P \cap F=\varnothing$.

For distributive nearlattices we have the following lemma which characterizes the generated filters and can be deduced from the results given in [9].

Lemma 1. Let $A$ be a distributive nearlattice. Let $X \subseteq A$ be a non-empty subset. Then

$$
F(X)=\left\{a \in A: \exists x_{1}, \ldots, x_{n} \in[X) \exists x_{1} \wedge \ldots \wedge x_{n}\left(x_{1} \wedge \ldots \wedge x_{n}=a\right)\right\} .
$$

A filter $H$ is said to be finitely generated if $H=F(X)$ for some finite non-empty subset $X$ of $A$. We will denote by $\mathrm{Fi}_{f}(A)$ the set of all finitely generated filters of $A$.

Recall that if $A$ is a distributive nearlattice, then $\langle\operatorname{Fi}(A), \underline{\vee}, \bar{\wedge},\{1\}, A\rangle$ is a bounded distributive lattice, where the least element is $\{1\}$, the greatest element is $A$, and for all $G, H \in \operatorname{Fi}(A)$ we have that $G \vee H=F(G \cup H)$ and $G \bar{\wedge} H=G \cap H$.

Theorem 3 ([4,9]). Let A be a nearlattice. The following conditions are equivalent:

(1) A is distributive.

(2) $\langle\operatorname{Fi}(A), \underline{\vee}, \bar{\lambda},\{1\}, A\rangle$ is a bounded distributive lattice.

(3) $\left\langle\mathrm{Fi}_{f}(A), \underline{\vee}, \pi,\{1\}, A\right\rangle$ is a bounded distributive lattice.

A function $h: A \rightarrow B$ between distributive nearlattices is a semi-homomorphism if $h(1)=1$ and $h(a \vee b)=h(a) \vee h(b)$ for all $a, b \in A$. A homomorphism is a semi-homomorphism $h$ such that for all $a, b \in A$, if $a \wedge b$ exists, then $h(a \wedge b)=h(a) \wedge h(b)$. In [3] it was shown that there exists a duality between semi-homomorphisms of distributive nearlattices and certain binary relations. 


\section{RELATIVE ANNIHILATORS}

In this section we will develop new characterizations of the distributivity of a nearlattice through relative annihilators and relative maximal ideals.

Definition 3. Let $A$ be a semilattice. For $a, b \in A$, the annihilator of a relative to $b$ is the set

$$
a \circ b=\{x \in A: b \leq x \vee a\} .
$$

Let $A$ be a semilattice. Let $a, b \in A, I \in \operatorname{Id}(A)$ and $F \in \operatorname{Fi}(A)$. We introduce the following subsets of $A$ :

$$
\begin{aligned}
& I \circ b=\{x \in A: \exists i \in I(b \leq x \vee i)\}, \\
& a \circ F=\{x \in A: \exists f \in F(f \leq x \vee a)\} .
\end{aligned}
$$

Let $X, Y \subseteq A$. We denote by $X \circ Y$ the set

$$
X \circ Y=\bigcup\{a \circ b:(a, b) \in X \times Y\} .
$$

Remark 1. Note that $a \circ b=(a] \circ b=a \circ[b)=(a] \circ[b)$ for all $a, b \in A$.

The following theorem characterizes distributive nearlattices.

Theorem 4. Let A be a nearlattice. The following conditions are equivalent:

(1) A is distributive.

(2) $a \circ b \in \operatorname{Fi}(A)$ for all $a, b \in A$.

(3) $I \circ b \in \operatorname{Fi}(A)$ for all $I \in \operatorname{Id}(A)$ and $b \in A$.

(4) $a \circ F \in \operatorname{Fi}(A)$ for all $F \in \operatorname{Fi}(A)$ and $a \in A$.

(5) $I \circ F \in \operatorname{Fi}(A)$ for all $I \in \operatorname{Id}(A)$ and $F \in \operatorname{Fi}(A)$.

Proof. (1) $\Rightarrow$ (2) It is obvious that $1 \in a \circ b$. Let $x, y \in A$ such that $x \leq y$ and $x \in a \circ b$. Then, $x \vee a \leq y \vee a$ and $b \leq x \vee a$. So, $b \leq y \vee a$ and $y \in a \circ b$. Let $x, y \in a \circ b$ such that $x \wedge y$ exists. Then, $b \leq x \vee a$ and $b \leq y \vee a$, i.e., $x \vee a, y \vee a \in$ $[b)$. Since $[b)$ is a bounded distributive lattice, $b \leq(x \vee a) \wedge_{b}(y \vee a)=(x \wedge y) \vee a$. Then, $x \wedge y \in a \circ b$ and $a \circ b \in \operatorname{Fi}(A)$.

(2) $\Rightarrow$ (3) Let $b \in A$ and $I \in \operatorname{Id}(A)$. We note that $i \circ b \subseteq I \circ b$ for all $i \in I$. It is easy to prove that $1 \in I \circ b$ and that $I \circ b$ is increasing. Let $x, y \in I \circ b$ such that $x \wedge y$ exists. Then there exist $i_{1}, i_{2} \in I$ such that $b \leq x \vee i_{1}$ and $b \leq y \vee i_{2}$. Let $i=i_{1} \vee i_{2} \in I$. So, $b \leq x \vee i$ and $b \leq y \vee i$. Since $x, y \in i \circ b$ and $i \circ b \in \operatorname{Fi}(A)$, we have that $x \wedge y \in i \circ b \subseteq I \circ b$. Therefore, $I \circ b \in \operatorname{Fi}(A)$.

(3) $\Rightarrow$ (4) Let $a \in A$ and $F \in \operatorname{Fi}(A)$. It follows easily that $1 \in a \circ F$ and that $a \circ F$ is increasing. Let $x, y \in a \circ F$ such that $x \wedge y$ exists. Then there exist $f_{1}, f_{2} \in F$ such that $f_{1} \leq x \vee a$ and $f_{2} \leq y \vee a$. So, $x \vee a, y \vee a \in F$. Since $x \vee a, y \vee a \in[a)$, $(x \vee a) \wedge_{a}(y \vee a)$ exists and $(x \vee a) \wedge_{a}(y \vee a) \in F$. As $(x \vee a) \wedge a(y \vee a) \leq x \vee a$ and $(x \vee a) \wedge_{a}(y \vee a) \leq y \vee a$, we have $x, y \in a \circ\left((x \vee a) \wedge_{a}(y \vee a)\right)$. By Remark 1 , $a \circ\left((x \vee a) \wedge_{a}(y \vee a)\right)=(a] \circ\left((x \vee a) \wedge_{a}(y \vee a)\right)$ and by hypothesis $(a] \circ\left((x \vee a) \wedge_{a}\right.$ 
$(y \vee a)) \in \operatorname{Fi}(A)$. Then $x \wedge y \in a \circ\left((x \vee a) \wedge_{a}(y \vee a)\right)$, but as $(x \vee a) \wedge_{a}(y \vee a) \in F$ we have that $x \wedge y \in a \circ F$. So, $a \circ F \in \operatorname{Fi}(A)$.

(4) $\Rightarrow(5)$ Let $I \in \operatorname{Id}(A)$ and $F \in \operatorname{Fi}(A)$. It is easy to see that $1 \in I \circ F$ and that $I \circ F$ is increasing. Let $x, y \in I \circ F$ such that $x \wedge y$ exists. Then there exist $\left(i_{1}, f_{1}\right),\left(i_{2}, f_{2}\right) \in I \times F$ such that $x \in i_{1} \circ f_{1}$ and $y \in i_{2} \circ f_{2}$, i.e., $f_{1} \leq x \vee i_{1}$ and $f_{2} \leq$ $y \vee i_{2}$. Let $i=i_{1} \vee i_{2} \in I$. On the other hand, $x \vee f_{1}, y \vee f_{2} \in F$ and $\left(x \vee f_{1}\right) \wedge x \wedge y$ $\left(y \vee f_{2}\right)$ exists in $[x \wedge y)$. It follows that $\left(x \vee f_{1}\right) \wedge x \wedge y\left(y \vee f_{2}\right) \in F$. We consider $i \circ F$. We note that $\left(x \vee f_{1}\right) \wedge_{x \wedge y}\left(y \vee f_{2}\right) \leq x \vee i$ and $\left(x \vee f_{1}\right) \wedge_{x \wedge y}\left(y \vee f_{2}\right) \leq y \vee i$. So, $x, y \in i \circ F$. By hypothesis $i \circ F \in \operatorname{Fi}(A)$ and $x \wedge y \in i \circ F$, i.e., there exists $f \in F$ such that $f \leq(x \wedge y) \vee i$. Then $x \wedge y \in i \circ f$ and $x \wedge y \in I \circ F$. Thus, $I \circ F \in \operatorname{Fi}(A)$.

(5) $\Rightarrow(1)$ Let $a \in A$ and $x, y, z \in[a)$. We know that the inequality $x \vee(y \wedge z) \leq$ $(x \vee y) \wedge(x \vee z)$ always holds. We prove the other inequality. As $(x \vee y) \wedge(x \vee z) \leq$ $y \vee x$ and $(x \vee y) \wedge(x \vee z) \leq z \vee x$ then $y, z \in x \circ((x \vee y) \wedge(x \vee z))$. By Remark 1 , $x \circ((x \vee y) \wedge(x \vee z))=(x] \circ[(x \vee y) \wedge(x \vee z))$ and by hypothesis $(x] \circ[(x \vee y) \wedge$ $(x \vee z)) \in \operatorname{Fi}(A)$. So, $y \wedge z \in(x] \circ[(x \vee y) \wedge(x \vee z))$, i.e., there exist $i \in(x]$ and $f \in[(x \vee y) \wedge(x \vee z))$ such that $y \wedge z \in i \circ f$. So, $f \leq(y \wedge z) \vee i$. It follows that $(x \vee y) \wedge(x \vee z) \leq x \vee(y \wedge z)$ and $[a)$ is a bounded distributive lattice.

In lattice theory, a lattice is distributive if and only if every proper ideal is an intersection of prime ideals. Here we present a generalization of this characterization.

Theorem 5. Let A be a nearlattice. The following conditions are equivalent:

(1) A is distributive.

(2) Every proper ideal of $A$ is an intersection of prime ideals.

Proof. (1) $\Rightarrow$ (2) See Corollary 2.9 of [3].

(2) $\Rightarrow$ (1) Let $a, b \in A$. We prove that $a \circ b \in \operatorname{Fi}(A)$. It is easy to see that $1 \in a \circ b$ and that $a \circ b$ is increasing. Let $x, y \in a \circ b$ such that $x \wedge y$ exists. Let $Q=((x \wedge y) \vee$ $a]$ and suppose that $b \notin Q$. So, $Q$ is a proper ideal and by hypothesis we have that $Q=\bigcap\{P \in X(A): Q \subseteq P\}$. Then there exists $P \in X(A)$ such that $(x \wedge y) \vee a \in P$ and $b \notin P$. So, $x \wedge y \in P$ and $a \in P$. As $P$ is prime, $x \in P$ or $y \in P$. Suppose that $x \in P$. Then $x \vee a \in P$ and since $x \in a \circ b$, i.e., $b \leq x \vee a$, we have that $b \in P$ which is a contradiction. The same reasoning applies when $y \in P$. Then $b \in Q$ and $b \leq(x \wedge y) \vee a$. Therefore, $x \wedge y \in a \circ b$ and $a \circ b \in \operatorname{Fi}(A)$. It follows from Theorem 4 that $A$ is distributive.

We study a new characterization of distributive nearlattices in terms of the notion of relative maximal ideal with respect to a set.

Definition 4. Let $A$ be a semilattice. Let $S$ be an increasing subset of $A$. An ideal $I$ of $A$ is called a relative maximal ideal with respect to $S$, when $I$ is maximal among all the ideals which are disjoint to $S$. 
Lemma 2. Let $A$ be a semilattice. Let $I \in \operatorname{Id}(A)$ and $F \in \operatorname{Fi}(A)$. Then $I$ is a relative maximal ideal with respect to $F$ if and only if $(H \circ F) \cap I \neq \varnothing$ for all $H \in \operatorname{Id}(A)$ such that $H \nsubseteq I$.

Proof. Suppose that $I$ is a relative maximal ideal with respect to $F$. Let $H \in \operatorname{Id}(A)$ such that $H \nsubseteq I$. We consider the ideal $I \vee H$. Since $I$ is a relative maximal ideal with respect to $F$ and $I \subseteq I \vee H$ then $(I \vee H) \cap F \neq \varnothing$, i.e., there exist $f \in F, i \in I$ and $h \in H$ such that $f \leq i \vee h$. So, $i \in h \circ f$ and $i \in H \circ F$. Therefore, $(H \circ F) \cap I \neq$ $\varnothing$.

Assume that $(H \circ F) \cap I \neq \varnothing$ for all $H \in \operatorname{Id}(A)$ such that $H \nsubseteq I$. Suppose that $I$ is not a relative maximal ideal with respect to $F$. Then there exists $J \in \operatorname{Id}(A)$ such that $I \subset J$ and $J \cap F=\varnothing$. Since $J \nsubseteq I$, by hypothesis we get $(J \circ F) \cap I \neq \varnothing$. Then there exist $i \in I$ and $(j, f) \in J \times F$ such that $i \in j \circ f$, i.e., $f \leq i \vee j$. As $i \in I \subset J$ and $i \vee j \in J$, we have that $f \in J$. So, $J \cap F \neq \varnothing$ which is a contradiction.

Theorem 6. Let A be a nearlattice. The following conditions are equivalent:

(1) A is distributive.

(2) Every relative maximal ideal I with respect to $a \circ b$ is prime for all $a, b \in A$.

Proof. (1) $\Rightarrow$ (2) Let $a, b \in A$ and $I \in \operatorname{Id}(A)$ such that $I$ is a relative maximal ideal with respect to $a \circ b$. We prove that $I$ is prime. Let $x, y \in A$ such that $x \wedge y$ exists and $x \wedge y \in I$. Suppose that $x \notin I$ and $y \notin I$. Let $I_{x}=I \vee(x]$ and $I_{y}=I \vee(y]$. Then $I_{x} \cap a \circ b \neq \varnothing$ and $I_{y} \cap a \circ b \neq \varnothing$, i.e., there exist $f_{1}, f_{2} \in a \circ b$ and $i_{1}, i_{2} \in I$ such that $f_{1} \leq x \vee i_{1}$ and $f_{2} \leq y \vee i_{2}$. Let $i=i_{1} \vee i_{2} \in I$. So, $x \vee i, y \vee i \in a \circ b$ and $(x \vee i) \wedge_{i}(y \vee i)$ exists in $[i)$. From Theorem 4, it follows that $a \circ b \in \operatorname{Fi}(A)$ and $(x \vee i) \wedge_{i}(y \vee i)=(x \wedge y) \vee i \in a \circ b$. On the other hand, as $I$ is an ideal, $(x \wedge y) \vee i \in I$. Thus, $I \cap a \circ b \neq \varnothing$ which is a contradiction. Then $I$ is prime.

(2) $\Rightarrow$ (1) By Theorem 4, it is sufficient to prove that $a \circ b \in \operatorname{Fi}(A)$. It is easy to see that $1 \in a \circ b$ and that $a \circ b$ is increasing. Let $x, y \in a \circ b$ such that $x \wedge y$ exists. Suppose that $x \wedge y \notin a \circ b$. Then $(x \wedge y] \cap a \circ b=\varnothing$. We consider the following family

$$
\mathcal{F}=\{I \in \operatorname{Id}(A):(x \wedge y] \subseteq I \text { and } I \cap a \circ b=\varnothing\} .
$$

So, $\mathcal{F} \neq \varnothing$. By Zorn's Lemma there exists a maximal element $M \in \mathcal{F}$. It is not difficult to show that $M$ is a relative maximal ideal with respect to $a \circ b$. So, $x \wedge y \in$ $M$ and by hypothesis $M$ is prime. Then $x \in M$ or $y \in M$. Thus, $M \cap a \circ b \neq \varnothing$ which is a contradiction. Therefore, $x \wedge y \in a \circ b$ and $a \circ b \in \operatorname{Fi}(A)$.

\section{NORMAL AND P-LINEAR NEARLATTICES}

Let $A$ be a semilattice and $a \in A$. From Definition 3 we have the following relative annihilator

$$
a^{\top}=a \circ 1=\{x \in A: x \vee a=1\},
$$

called the annihilator of $a$. We have the following result. 
Lemma 3. Let $A$ be a distributive nearlattice. Let $a, b \in A$ and $I \in \operatorname{Id}(A)$. Then

(1) $I \cap a \circ b=\varnothing$ if and only if there exists $Q \in X(A)$ such that $I \subseteq Q, a \in Q$ and $b \notin Q$.

(2) $I \cap a^{\top}=\varnothing$ if and only if there exists $Q \in X(A)$ such that $I \subseteq Q$ and $a \in Q$.

(3) $I \cap a^{\top}=\varnothing$ if and only if there exists $U \in \operatorname{Idm}(A)$ such that $I \subseteq U$ and $a \in U$.

(4) $U \in \operatorname{Idm}(A)$ if and only if for all $a \in A, a \notin U$ if and only if $U \cap a^{\top} \neq \varnothing$.

Proof. (1) Let $J \in \operatorname{Id}(A)$ such that $J \cap a \circ b=\varnothing$. Let $H=I(J \cup\{a\})$. We prove that $H \cap[b)=\varnothing$. If $x \in H \cap[b)$ then there exists $j \in J$ such that $x \leq j \vee a$ and $b \leq x$. So, $b \leq j \vee a$ and $j \in a \circ b$ which is a contradiction. Then $H \cap[b)=\varnothing$ and by Theorem 2 there exists $Q \in X(A)$ such that $J \subseteq Q, a \in Q$ and $b \notin Q$.

The other direction is immediate.

(2) It follows from (1).

(3) If $I \cap a^{\top}=\varnothing$ then there exists $Q \in X(A)$ such that $I \subseteq Q$ and $a \in Q$. We consider the family

$$
\mathcal{Z}=\{R \in \operatorname{Id}(A)-\{A\}: I \subseteq R \text { and } a \in R\} .
$$

So, $\mathcal{Z} \neq \varnothing$ because $Q \in \mathcal{Z}$. Then, by Zorn's Lemma, there exists a maximal element $U \in \mathcal{Z}$. It is clear that $U$ is proper. We prove that $U$ is a maximal ideal. Let $b \in A$ such that $b \notin U$. If $U \cap b^{\top}=\varnothing$ then $H=I(U \cup\{b\})$ is a proper ideal. Otherwise, if $1 \in H$ then there exists $p \in U$ such that $p \vee b=1$, i.e., $p \in U \cap b^{\top}$ which is a contradiction. So $U \subset H$ and $H \in \mathcal{Z}$, which is a contradiction because $U$ is maximal. Then $U \cap b^{\top} \neq \varnothing$ and there exists $c \in U$ such that $c \vee b=1$. Therefore, $H=A$ and $U$ is maximal.

Suppose that $I \cap a^{\top} \neq \varnothing$. Then there exists $i \in I$ such that $i \vee a=1$. So, there exists $U \in \operatorname{Idm}(A)$ such that $I \subseteq Q$ and $a \in Q$. Then $i \vee a=1 \in U$, which is a contradiction because $U$ is maximal.

(4) Let $U \in \operatorname{Idm}(A)$. Suppose that $a \notin U$. As $U$ is maximal, $I(U \cup\{a\})=A$. Then $1 \in I(U \cup\{a\})$, i.e., there exists $p \in U$ such that $p \vee a=1$. So, $p \in a^{\top}$ and $U \cap a^{\top} \neq \varnothing$.

If $U \cap a^{\top} \neq \varnothing$ and $a \in U$ then there exists $p \in U$ such that $p \vee a=1$. Thus, $1 \in U$ which is a contradiction.

Conversely, let $I \in \operatorname{Id}(A)$ such that $U \subset I$. Then there exists $a \in I$ such that $a \notin U$. So, $U \cap a^{\top} \neq \varnothing$, i.e., there exists $p \in U$ such that $p \vee a=1$. Since $U \subset I$, we have that $p \in I$ and $a \vee p=1 \in I$. Therefore, $I=A$ and $U$ is maximal.

We recall that a bounded distributive lattice is normal if each prime ideal contains a unique minimal prime ideal. This concept was introduced by Cornish in [8] and extended to the class of distributive semilattices in [13]. Now, we introduce a generalization of this notion.

Definition 5. Let $A$ be a distributive nearlattice. We say that $A$ is normal if each prime ideal is contained in a unique maximal ideal. 
We say that $A$ is $p$-linear if the family of prime ideals which contain a prime ideal is a chain.

Remark 2. We note that every normal nearlattice is p-linear.

Remark 3. If $A$ is a bounded distributive lattice then the Definition 5 is equivalent to saying that every prime filter contains a unique minimal filter, which is a concept dual to the definition given by Cornish in [8].

The following results characterize normal nearlattices through annihilators.

Lemma 4. Let $A$ be a distributive nearlattice. The following conditions are equivalent:

(1) A is normal.

(2) For every $P \in X(A)$ and for all $a, b \in A$ with $a \vee b=1, P \cap a^{\top} \neq \varnothing$ or $P \cap b^{\top} \neq \varnothing$.

Proof. (1) $\Rightarrow(2)$ Let $P \in X(A)$ and $a, b \in A$ such that $a \vee b=1$. Suppose that $P \cap a^{\top}=\varnothing$ and $P \cap b^{\top}=\varnothing$. So, by Lemma 3, there exist $U_{1}, U_{2} \in \operatorname{Idm}(A)$ such that $P \subseteq U_{1}, P \subseteq U_{2}, a \in U_{1}$ and $b \in U_{2}$. Since $A$ is normal, $U_{1}=U_{2}$. Then $a, b \in U_{1}$, but $a \vee b=1 \in U_{1}$ which is a contradiction. Thus, $P \cap a^{\top} \neq \varnothing$ or $P \cap b^{\top} \neq \varnothing$.

(2) $\Rightarrow$ (1) Let $P \in X(A)$ and $U_{1}, U_{2} \in \operatorname{Idm}(A)$ such that $P \subseteq U_{1}$ and $P \subseteq U_{2}$. If $U_{1} \neq U_{2}$ then there exists $a \in U_{1}$ such that $a \notin U_{2}$. As $U_{2}$ is maximal, $I\left(U_{2} \cup\right.$ $\{a\})=A$. Then $1 \in I\left(U_{2} \cup\{a\}\right)$, i.e., there exists $b \in U_{2}$ such that $a \vee b=1$. On the other hand, by Lemma 3, $P \cap a^{\top}=\varnothing$ and $P \cap b^{\top}=\varnothing$, which contradicts the assumption.

Lemma 5. Let $A$ be a distributive nearlattice. The following conditions are equivalent:

(1) A is normal.

(2) For all $a, b \in A,(a \vee b)^{\top}=F\left(a^{\top} \cup b^{\top}\right)$.

(3) For all $a, b \in A$ with $a \vee b=1, F\left(a^{\top} \cup b^{\top}\right)=A$.

Proof. (1) $\Rightarrow(2)$ Let $a, b \in A$. Note that the inclusion $F\left(a^{\top} \cup b^{\top}\right) \subseteq(a \vee b)^{\top}$ always holds. Let us prove the other inclusion. Suppose that there exists $x \in(a \vee b)^{\top}$ such that $x \notin F\left(a^{\top} \cup b^{\top}\right)$. So, by Theorem 2, there exists $P \in X(A)$ such that $x \in P$ and $P \cap F\left(a^{\top} \cup b^{\top}\right)=\varnothing$. Since $a^{\top}, b^{\top} \subseteq F\left(a^{\top} \cup b^{\top}\right)$, we have that $P \cap a^{\top}=\varnothing$ and $P \cap b^{\top}=\varnothing$. Then, by Lemma 3, there exist $U_{1}, U_{2} \in \operatorname{Idm}(A)$ such that $P \subseteq U_{1}, P \subseteq$ $U_{2}, a \in U_{1}$ and $b \in U_{2}$. As $A$ is normal, $U_{1}=U_{2}$ and $a, b \in U_{1}$. Also, $x \in U_{1}$. Then $x \vee(a \vee b)=1 \in U_{1}$ which is a contradiction. Therefore, $(a \vee b)^{\top}=F\left(a^{\top} \cup b^{\top}\right)$.

(2) $\Rightarrow$ (3) It is immediate.

(3) $\Rightarrow(1)$ Let $P \in X(A)$ and $U_{1}, U_{2} \in \operatorname{Idm}(A)$ such that $P \subseteq U_{1}$ and $P \subseteq U_{2}$. Suppose that there exists $a \in U_{1}$ such that $a \notin U_{2}$. Since $U_{2}$ is maximal, there exists $b \in U_{2}$ such that $a \vee b=1$. Then $F\left(a^{\top} \cup b^{\top}\right)=A$. Let $x \in P$. Hence, $x \in F\left(a^{\top} \cup b^{\top}\right)$ and there exist $x_{1}, \ldots, x_{n} \in a^{\top} \cup b^{\top}$ such that $x_{1} \wedge \ldots \wedge x_{n}$ exists and $x_{1} \wedge \ldots \wedge x_{n}=x$. 
So, $x_{1} \wedge \ldots \wedge x_{n} \in P$ and by the primality of $P$ there exists $x_{i} \in\left\{x_{1}, \ldots, x_{n}\right\}$ such that $x_{i} \in P$. If $x_{i} \in a^{\top}$ then $x_{i} \in U_{1}$ and $x_{i} \vee a=1 \in U_{1}$, which is a contradiction because $U_{1}$ is proper. If $x_{i} \in b^{\top}$, we arrive at a contradiction. Thus, $U_{1} \subseteq U_{2}$ and consequently $U_{1}=U_{2}$. Therefore, $A$ is normal.

Corollary 1. Let $A$ be a distributive nearlattice. The following conditions are equivalent:

(1) A is normal.

(2) The application $\rho: A \rightarrow \operatorname{Fi}(A)$ defined by $\rho(a)=a^{\top}$ is a homomorphism of distributive nearlattices.

Proof. It follows from Lemma 5.

Theorem 7. Let $A$ be a distributive nearlattice. The following conditions are equivalent:

(1) A is p-linear.

(2) For every $P \in X(A)$ and for all $a, b \in A, P \cap a \circ b \neq \varnothing$ or $P \cap b \circ a \neq \varnothing$.

(3) For all $a, b \in A, F((a \circ b) \cup(b \circ a))=A$.

Proof. (1) $\Rightarrow$ (2) Let $P \in X(A)$ and $a, b \in A$. Assume that $P \cap a \circ b=\varnothing$ and $P \cap b \circ a=\varnothing$. By Lemma 3 there exist $Q_{1}, Q_{2} \in X(A)$ such that $P \subseteq Q_{1}, a \in Q_{1}$, $b \notin Q_{1}, P \subseteq Q_{2}, b \in Q_{2}$, and $a \notin Q_{2}$. As $A$ is p-linear, $Q_{1} \subseteq Q_{2}$ or $Q_{2} \subseteq Q_{1}$. If $Q_{1} \subseteq Q_{2}$ then $a \in Q_{2}$, which is impossible. If $Q_{2} \subseteq Q_{1}$, then $b \in Q_{1}$ which is a contradiction. Thus, $P \cap a \circ b \neq \varnothing$ or $P \cap b \circ a \neq \varnothing$.

(2) $\Rightarrow$ (3) Suppose that there exist $a, b \in A$ such that $F((a \circ b) \cup(b \circ a)) \neq A$. Then there exists $c \in A$ such that $c \notin F((a \circ b) \cup(b \circ a))$. Since $F((a \circ b) \cup(b \circ a)) \in \operatorname{Fi}(A)$, by Theorem 2 there exists $P \in X(A)$ such that $c \in P$ and $P \cap F((a \circ b) \cup(b \circ a))=$ $\varnothing$. So, $P \cap a \circ b=\varnothing$ and $P \cap b \circ a=\varnothing$ which is impossible. Thus, $F((a \circ b) \cup(b \circ$ a)) $=A$.

(3) $\Rightarrow$ (1) Let $P, Q_{1}, Q_{2} \in X(A)$ such that $P \subseteq Q_{1}$ and $P \subseteq Q_{2}$. If $Q_{1}$ and $Q_{2}$ are incomparable, then there exist $a, b \in A$ such that $a \in Q_{1}-Q_{2}$ and $b \in Q_{2}-Q_{1}$. Let $x \in P$. Since $A=F((a \circ b) \cup(b \circ a))$, there exist $x_{1}, \ldots, x_{n} \in(a \circ b) \cup(b \circ a)$ such that $x_{1} \wedge \ldots \wedge x_{n}$ exists and $x_{1} \wedge \ldots \wedge x_{n}=x$. So, $x_{1} \wedge \ldots \wedge x_{n} \in P$ and by the primality of $P$ there exists $x_{i} \in\left\{x_{1}, \ldots, x_{n}\right\}$ such that $x_{i} \in P$. If $x_{i} \in a \circ b$, then $b \leq x_{i} \vee a$. As $x_{i}, a \in Q_{1}$, we have $x_{i} \vee a \in Q_{1}$ and $b \in Q_{1}$ which is a contradiction. Similarly, if $x_{i} \in b \circ a$ we arrive at a contradiction. Thus, $Q_{1}$ and $Q_{2}$ are comparable and $A$ is p-linear.

\section{THE LATTICE OF FILTERS}

In this section we study the structure of the lattice of filters of a distributive nearlattice. Recall that a Heyting algebra is an algebra $\langle A, \vee, \wedge, \Rightarrow, 0,1\rangle$ of type $(2,2,2,0,0)$ such that $\langle A, \vee, \wedge, 0,1\rangle$ is a bounded distributive lattice and the operation $\Rightarrow$ satisfies 
the following condition: $a \wedge b \leq c$ if and only if $a \leq b \Rightarrow c$ for all $a, b, c \in A$. The pseudocomplement of an element $a \in A$ is the element $a^{*}=a \Rightarrow 0$.

Let $A$ be a distributive nearlattice. For each pair $F, H \in \operatorname{Fi}(A)$ let us define the subset $F \triangleright H$ of $A$ as follows:

$$
F \triangleright H=\{a \in A:[a) \cap F \subseteq H\} .
$$

Theorem 8. Let $A$ be a distributive nearlattice. Let $F, H \in \operatorname{Fi}(A)$. Then

(1) $F \triangleright H \in \operatorname{Fi}(A)$.

(2) $F \triangleright H=\{a \in A: \forall f \in F \exists h \in H(h \leq a \vee f)\}$.

(3) The structure $\langle\operatorname{Fi}(A), \underline{\vee}, \bar{\wedge}, \triangleright,\{1\}, A\rangle$ is a Heyting algebra.

Proof. (1) Let $F, H \in \operatorname{Fi}(A)$. We prove that $F \triangleright H \in \operatorname{Fi}(A)$. Since [1) $\cap F=$ $\{1\} \subseteq H$, then $1 \in F \triangleright H$. Let $a, b \in A$ such that $a \leq b$ and $a \in F \triangleright H$. Thus, $[b] \subseteq[a)$ and $[a) \cap F \subseteq H$. It follows that $[b] \cap F \subseteq H$, i.e., $b \in F \triangleright H$. Let $a, b \in F \triangleright H$ and suppose that $a \wedge b$ exists. By Theorem 3, the lattice $\operatorname{Fi}(A)$ is distributive and

$$
\begin{aligned}
{[a \wedge b) \cap F } & =([a) \vee[b)) \cap F \\
& =([a) \cap F) \underline{\vee}([b) \cap F) \\
& \subseteq H .
\end{aligned}
$$

Therefore, $a \wedge b \in F \triangleright H$ and $F \triangleright H \in \operatorname{Fi}(A)$.

(2) Let $F, H \in \operatorname{Fi}(A)$. Let $X=\{a \in A: \forall f \in F \exists h \in H(h \leq a \vee f)\}$ and $a \in X$. We prove that $a \in F \triangleright H$, i.e., $[a) \cap F \subseteq H$. If $x \in[a) \cap F$, then $a \leq x$ and $x \in F$. Since $a \in X$ and $x \in F$, there exists $h \in H$ such that $h \leq a \vee x=x$. As $H$ is a filter, $x \in H$. So, $a \in F \triangleright H$. Conversely, let $a \in F \triangleright H$ and $f \in F$. Then $a \vee f \in[a) \cap F$ and by hypothesis, $a \vee f \in H$. It follows that $a \in X$. Therefore, $F \triangleright H=X$.

(3) By Theorem $3,\langle\operatorname{Fi}(A), \underline{\vee}, \bar{\wedge},\{1\}, A\rangle$ is a bounded distributive lattice. Let $F, G, H \in \operatorname{Fi}(A)$. We prove that $F \cap G \subseteq H$ if and only if $F \subseteq G \triangleright H$. Suppose that $F \cap G \subseteq H$ and let $a \in F$. If $x \in[a) \cap G$, then $a \leq x$ and $x \in G$. Therefore $x \in F \cap G$ and by hypothesis $x \in H$, i.e., $F \subseteq G \triangleright H$. Conversely, let $x \in F \cap G$. By hypothesis $F \subseteq G \triangleright H$, then $x \in G \triangleright H$. As $[x) \cap G \subseteq H$, we have that $x \in H$.

Remark 4. As a particular case, we have $a \circ b=[a) \triangleright[b)$. Indeed

$$
\begin{aligned}
& x \in a \circ b \quad \text { iff } \quad b \leq x \vee a \quad \text { iff } \quad[x \vee a) \subseteq[b) \\
& \text { iff }[x)^{\circ} \cap[a] \subseteq[b] \text { iff } \quad x \in[a) \triangleright[b) \text {. }
\end{aligned}
$$

Then we can write the annihilator of $a$ relative to $b$ in terms of the binary operation $\triangleright$.

Note that if $F \in \operatorname{Fi}(A)$, then $F^{*}=F \triangleright\{1\}=\{a \in A:[a) \cap F=\{1\}\}$. The following result describes the filter $F^{*}$ in a different manner.

Proposition 2. Let $A$ be a distributive nearlattice. Then for every $F \in \operatorname{Fi}(A)$,

$$
F^{*}=\{a \in A: \forall f \in F(a \vee f=1)\} .
$$


Proof. Let $C=\{a \in A: \forall f \in F(a \vee f=1)\}$ and $a \in C$. We prove that $[a) \cap F=$ $\{1\}$. Let $x \in A$ such that $a \leq x$ and $x \in F$. Then $x=a \vee x=1$. Thus, $a \in F^{*}$. Conversely, let $a \in F^{*}$. Then $[a) \cap F=\{1\}$. Since $a \leq a \vee f$ and $f \leq a \vee f$ for every $f \in F$, and as $F$ is a filter, $a \vee f \in[a) \cap F$. So, $a \vee f=1$ for each $f \in F$. Therefore, $a \in C$ and $F^{*}=C$.

Remark 5. We note that $[a)^{*}=\{x \in A: x \vee a=1\}$, i.e., $a^{\top}=[a)^{*}$.

We prove that the pseudocomplement of a subset $X$ is the pseudocomplement of the filter generated by $X$. This result was proved for Tarski algebras in [5].

Theorem 9. Let $A$ be a distributive nearlattice. Then for every subset $X \subseteq A$, we have $X^{*}=F(X)^{*}$.

Proof. Since $X \subseteq F(X)$, we have that $F(X)^{*} \subseteq X^{*}$. Conversely, let $x \in X^{*}$. We prove that for every $a \in F(X), x \vee a=1$. Suppose that there exists $a \in F(X)$ such that $x \vee a \neq 1$. Then there exist $x_{1}, \ldots, x_{n} \in[X)$ such that $x_{1} \wedge \ldots \wedge x_{n}$ exists and $x_{1} \wedge \ldots \wedge x_{n}=a$. So, there exist $y_{1}, \ldots, y_{n} \in X$ such that $y_{i} \leq x_{i}$ for all $i \in$ $\{1, \ldots, n\}$. As $x \in X^{*}, x \vee y_{i}=1$ for all $y_{i} \in\left\{y_{1}, \ldots, y_{n}\right\}$. Then $x \vee x_{i}=1$ for all $x_{i} \in\left\{x_{1}, \ldots, x_{n}\right\}$. Since $x \vee a \neq 1$, by Theorem 2, there exists $P \in X(A)$ such that $x \vee a \in P$ and $1 \notin P$. Then $x_{1} \wedge \ldots \wedge x_{n} \in P$ and as $P$ is a prime ideal, there exists $x_{i} \in\left\{x_{1}, \ldots, x_{n}\right\}$ such that $x_{i} \in P$. On the other hand, $x \vee a \in P$ and $x \in P$. So, $x \vee x_{i}=1 \in P$ which is a contradiction. Thus, $x \vee a=1$ for all $a \in F(X)$ and consequently $x \in F(X)^{*}$.

\section{SEMI-HOMOMORPHISMS PRESERVING ANNIHILATORS}

Our next aim is to study a particular class of semi-homomorphisms: semi-homomorphisms preserving annihilators. We give some characterizations in terms of prime and maximal ideals.

Definition 6. Let $A, B$ be two distributive nearlattices and let $h: A \rightarrow B$ be a semihomomorphism. We say that $h$ is a semi-homomorphism preserving annihilators, or T-semi-homomorphism, if $F\left(h\left(a^{\top}\right)\right)=h(a)^{\top}$ for all $a \in A$.

Remark 6. We note that $F\left(h\left(a^{\top}\right)\right) \subseteq h(a)^{\top}$ for all $a \in A$. If $x \in F\left(h\left(a^{\top}\right)\right)$ then there exist $x_{1}, \ldots, x_{n} \in\left[h\left(a^{\top}\right)\right)$ such that $x_{1} \wedge \ldots \wedge x_{n}$ exists and $x_{1} \wedge \ldots \wedge x_{n}=x$. As $x_{1}, \ldots, x_{n} \in\left[h\left(a^{\top}\right)\right)$, there exist $y_{1}, \ldots, y_{n} \in h\left(a^{\top}\right)$ such that $y_{i} \leq x_{i}$ for all $i \in$ $\{1, \ldots, n\}$. So, there exist $t_{1}, \ldots, t_{n} \in a^{\top}$ such that $h\left(t_{i}\right)=y_{i}$ for $1 \leq i \leq n$. Thus, $t_{1} \vee$ $a=\cdots=t_{n} \vee a=1$ and since $h$ is a semi-homomorphism, we have that $y_{1} \vee h(a)=$ $\cdots=y_{n} \vee h(a)=1$. Then $x \vee h(a)=\left[\left(y_{1} \vee x_{1}\right) \wedge \ldots \wedge\left(y_{n} \vee x_{n}\right)\right] \vee h(a)$. As $[x)$ is a bounded distributive lattice, $x \vee h(a)=\left(y_{1} \vee x_{1} \vee h(a)\right) \wedge \ldots \wedge\left(y_{n} \vee x_{n} \vee h(a)\right)=1$. Therefore, $x \vee h(a)=1$ and $x \in h(a)^{\top}$.

Let $h: A \rightarrow B$ be a semi-homomorphism between distributive nearlattices. In general, $h^{-1}(P) \notin X(A)$ for each $P \in X(B)$. Now, we prove that if $P$ is maximal

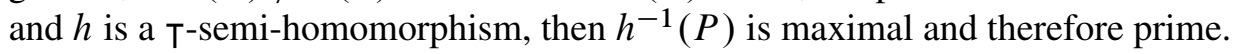


Lemma 6. Let $A, B$ be two distributive nearlattices and let $h: A \rightarrow B$ be a $\mathrm{T}^{-}$ semi-homomorphism. Then $h^{-1}(P) \in \operatorname{Idm}(A)$ for every $P \in \operatorname{Idm}(B)$.

Proof. Let $P \in \operatorname{Idm}(B)$. Since $h$ is a semi-homomorphism, $h^{-1}(P)$ is an ideal. As $h(1)=1 \notin P$, we have that $h^{-1}(P)$ is proper. Let $a \in A$ such that $a \notin h^{-1}(P)$. Then $h(a) \notin P$ and as $P$ is maximal, by Lemma $3, P \cap h(a)^{\top} \neq \varnothing$. So, there exists $x \in A$ such that $x \in P \cap F\left(h\left(a^{\top}\right)\right) \neq \varnothing$, i.e., there exist $x_{1}, \ldots, x_{n} \in\left[h\left(a^{\top}\right)\right)$ such that $x_{1} \wedge \ldots \wedge x_{n}$ exists and $x_{1} \wedge \ldots \wedge x_{n}=x$. Thus, there exist $y_{1}, \ldots, y_{n} \in h\left(a^{\top}\right)$ such that $y_{i} \leq x_{i}$ for all $i \in\{1, \ldots, n\}$. It follows that there exist $t_{1}, \ldots, t_{n} \in a^{\top}$ such that $h\left(t_{i}\right)=y_{i}$ for $1 \leq i \leq n$. Then $t_{1} \vee a=\ldots=t_{n} \vee a=1$ and since $h$ is a semihomomorphism, we have that $y_{1} \vee h(a)=\ldots=y_{n} \vee h(a)=1$. As

$$
x=x_{1} \wedge \ldots \wedge x_{n}=\left(x_{1} \vee y_{1}\right) \wedge \ldots \wedge\left(x_{n} \vee y_{n}\right) \in P
$$

and $P$ is prime, there exists $i \in\{1, \ldots, n\}$ such that $x_{i} \vee y_{i} \in P$. So, $y_{i}=h\left(t_{i}\right) \in$ $P$, i.e., $t_{i} \in h^{-1}(P)$ and $h^{-1}(P) \cap a^{\top} \neq \varnothing$. Conversely, it is easy to prove that if $h^{-1}(P) \cap a^{\top} \neq \varnothing$, then $a \notin h^{-1}(P)$. Therefore, by Lemma $3, h^{-1}(P) \in \operatorname{Idm}(A)$.

Theorem 10. Let $A, B$ be two distributive nearlattices and let $h: A \rightarrow B$ be a semi-homomorphism. Then the following conditions are equivalent:

(1) $h$ is a T-semi-homomorphism.

(2) For all $P \in X(B)$ and for every $Q \in X(A)$ such that $h^{-1}(P) \subseteq Q$, there exists $D \in X(B)$ such that $P \subseteq D$ and $Q \subseteq h^{-1}(D)$.

(3) $\operatorname{Idm}(A) \cap\left[h^{-1}(P)\right) \subseteq h^{-1}[X(B) \cap[P)]$ for all $P \in X(B)$.

Proof. (1) $\Rightarrow$ (2) Let $P \in X(B)$ and $Q \in X(A)$ such that $h^{-1}(P) \subseteq Q$. Let us consider the ideal $H=I(P \cup h(Q))$. We note that $H$ is a proper ideal. Indeed, if we assume otherwise, there exists $p \in P$ and $q \in Q$ such that $p \vee h(q)=1$. So, $p \in h(q)^{\top}=F\left(h\left(q^{\top}\right)\right)$. Then, there exist $x_{1}, \ldots, x_{n} \in\left[h\left(q^{\top}\right)\right)$ such that $x_{1} \wedge \ldots \wedge x_{n}$ exists and $x_{1} \wedge \ldots \wedge x_{n}=p$. So, there exist $y_{1}, \ldots, y_{n} \in h\left(q^{\top}\right)$ such that $y_{i} \leq x_{i}$ for all $i \in\{1, \ldots, n\}$. It follows that there exist $t_{1}, \ldots, t_{n} \in q^{\top}$ such that $h\left(t_{i}\right)=y_{i}$ for $1 \leq i \leq$ $n$. Then $t_{1} \vee q=\ldots=t_{n} \vee q=1$ and since $h$ is a semi-homomorphism, we have that $y_{1} \vee h(q)=\ldots=y_{n} \vee h(q)=1$. As $x=x_{1} \wedge \ldots \wedge x_{n}=\left(x_{1} \vee y_{1}\right) \wedge \ldots \wedge\left(x_{n} \vee y_{n}\right) \in P$ and $P$ is prime, there exists $i \in\{1, \ldots, n\}$ such that $x_{i} \vee y_{i} \in P$. So, $y_{i}=h\left(t_{i}\right) \in P$, i.e., $t_{i} \in h^{-1}(P) \subseteq Q$ and since $q \in Q, t_{i} \vee q=1 \in Q$ which is a contradiction. Therefore, $H$ is a proper ideal and there exists $D \in X(B)$ such that $P \subseteq D$ and $Q \subseteq h^{-1}(D)$.

(2) $\Rightarrow(3)$ Let $P \in X(B)$ and $Q \in \operatorname{Idm}(A) \cap\left[h^{-1}(P)\right)$. Then $Q \in X(A)$ and $h^{-1}(P) \subseteq Q$. By hypothesis, there exists $D \in X(B)$ such that $P \subseteq D$ and $Q \subseteq$ $h^{-1}(D)$. Since $h^{-1}(D)$ is an ideal and $Q$ is maximal, $Q=h^{-1}(D)$. So, $D \in$ $h^{-1}[X(B) \cap[P)]$ and $\operatorname{Idm}(A) \cap\left[h^{-1}(P)\right) \subseteq h^{-1}[X(B) \cap[P)]$.

(3) $\Rightarrow$ (1) Let $a \in A$. We prove that $h(a)^{\top} \subseteq F\left(h\left(a^{\top}\right)\right)$. Suppose that there exists $x \in h(a)^{\top}$ such that $x \notin F\left(h\left(a^{\top}\right)\right)$. By Theorem 2, there exists $P \in X(A)$ such that 
$x \in P$ and $P \cap F\left(h\left(a^{\top}\right)\right)=\varnothing$. Then,

$$
P \cap h(a)^{\top} \neq \varnothing \text { and } P \cap h\left(a^{\top}\right)=\varnothing .
$$

Thus, $h^{-1}(P) \cap a^{\top}=\varnothing$ and $h^{-1}(P) \in \operatorname{Id}(A)$. By Lemma 3, there exists $U \in \operatorname{Idm}(A)$ such that $h^{-1}(P) \subseteq U$ and $a \in U$. So, we get that

$$
U \in \operatorname{Idm}(A) \cap\left[h^{-1}(P)\right) \subseteq h^{-1}[X(B) \cap[P)] .
$$

Then, there exists $D \in X(B)$ such that $P \subseteq D$ and $U=h^{-1}(D)$. Since $a \in U$, $h(a) \in D$ and $D \cap h(a)^{\top}=\varnothing$. On the other hand, $P \cap h(a)^{\top} \neq \varnothing$ and $P \subseteq D$. Thus, $D \cap h(a)^{\top} \neq \varnothing$ which is a contradiction. Therefore, $h(a)^{\top} \subseteq F\left(h\left(a^{\top}\right)\right)$ and $h$ is a T-semi-homomorphism.

It is possible to give another characterization of the $\mathrm{T}$-semi-homomorphisms in normal nearlattices.

Proposition 3. Let $A, B$ be two distributive nearlattices and let $h: A \rightarrow B$ be a semi-homomorphism. Suppose that $B$ is normal. Then $h$ is a T-semi-homomorphism if and only if:

(1) For all $P \in X(B)$ and for all $Q_{1}, Q_{2} \in \operatorname{Idm}(A)$, if $h^{-1}(P) \subseteq Q_{1} \cap Q_{2}$ then $Q_{1}=Q_{2}$

(2) $h^{-1}(P) \in \operatorname{Idm}(A)$ for every $P \in \operatorname{Idm}(B)$.

Proof. $\Rightarrow)$ Suppose that $h$ is a T-semi-homomorphism. By Lemma 6, we only need to prove (1). Let $P \in X(B)$ and $Q_{1}, Q_{2} \in \operatorname{Idm}(A)$ such that $h^{-1}(P) \subseteq Q_{1} \cap$ $Q_{2}$. Suppose that there exists $a \in Q_{1}$ such that $a \notin Q_{2}$. Since $Q_{2}$ is maximal, there exists $b \in Q_{2}$ such that $a \vee b=1$. Then $h(a \vee b)=h(a) \vee h(b)=h(1)=1$. As $B$ is normal, by Lemma 4 , we have that $P \cap h(a)^{\top} \neq \varnothing$ or $P \cap h(b)^{\top} \neq \varnothing$, and

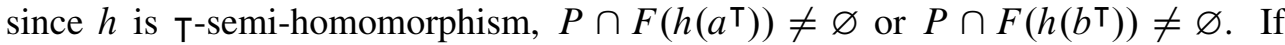
$P \cap F\left(h\left(a^{\top}\right)\right) \neq \varnothing$, then there exists $x \in P$ such that $x \in F\left(h\left(a^{\top}\right)\right)$, i.e., there exist $x_{1}, \ldots, x_{n} \in\left[h\left(a^{\top}\right)\right)$ such that $x_{1} \wedge \ldots \wedge x_{n}$ exists and $x_{1} \wedge \ldots \wedge x_{n}=x$. So, there exist $y_{1}, \ldots, y_{n} \in h\left(a^{\top}\right)$ such that $y_{i} \leq x_{i}$ for all $i \in\{1, \ldots, n\}$. It follows that there exist $t_{1}, \ldots, t_{n} \in a^{\top}$ such that $h\left(t_{i}\right)=y_{i}$ for $1 \leq i \leq n$. Then, $t_{1} \vee a=\ldots=t_{n} \vee a=1$ and since $h$ is a semi-homomorphism, we have that $y_{1} \vee h(a)=\ldots=y_{n} \vee h(a)=1$. As $x=x_{1} \wedge \ldots \wedge x_{n}=\left(x_{1} \vee y_{1}\right) \wedge \ldots \wedge\left(x_{n} \vee y_{n}\right) \in P$ and $P$ is prime, there exists $i \in\{1, \ldots, n\}$ such that $x_{i} \vee y_{i} \in P$. So, $y_{i}=h\left(t_{i}\right) \in P$ and $t_{i} \in h^{-1}(P) \subseteq Q_{1} \cap Q_{2}$. Since $a, t_{i} \in Q_{1}$, we have that $t_{i} \vee a=1 \in Q_{1}$, which is a contradiction because $Q_{1}$ is maximal. If $P \cap F\left(h\left(b^{\top}\right)\right) \neq \varnothing$, we get also a contradiction. Therefore, $Q_{1} \subseteq Q_{2}$ and consequently $Q_{1}=Q_{2}$.

$\Leftarrow)$ Let $a \in A$. We prove that $h(a)^{\top} \subseteq F\left(h\left(a^{\top}\right)\right)$. Suppose that there exists $x \in$ $h(a)^{\top}$ such that $x \notin F\left(h\left(a^{\top}\right)\right)$. Then there exists $P \in X(B)$ such that $x \in P$ and $P \cap F\left(h\left(a^{\top}\right)\right)=\varnothing$, i.e., $P \cap h(a)^{\top} \neq \varnothing$ and $P \cap F\left(h\left(a^{\top}\right)\right)=\varnothing$. Since $B$ is normal, there exists a unique $Q \in \operatorname{Idm}(B)$ such that $P \subseteq Q$. We note that $h(a) \notin Q$. Indeed, if $h(a) \in Q$ then $h(a)^{\top} \cap Q \neq \varnothing$ and there exists $x \in Q$ such that $h(a) \vee x=1 \in Q$, 
which is a contradiction. Since $a \notin h^{-1}(Q)$ and by (2) we have $h^{-1}(Q) \in \operatorname{Idm}(A)$, then, by Lemma $3, h^{-1}(Q) \cap a^{\top} \neq \varnothing$, i.e., $Q \cap F\left(h\left(a^{\top}\right)\right) \neq \varnothing$. On the other hand, since $P \cap F\left(h\left(a^{\top}\right)\right)=\varnothing$ then $h^{-1}(P) \cap a^{\top}=\varnothing$ and, by Lemma 3, there exists $U \in \operatorname{Idm}(A)$ such that $h^{-1}(P) \subseteq U$ and $a \in U$. Then $h^{-1}(P) \subseteq h^{-1}(Q) \cap U$ and by (1), we have $h^{-1}(Q)=U$, which is a contradiction because $a \in U$ and $a \notin h^{-1}(Q)$. Therefore, $h(a)^{\top}=F\left(h\left(a^{\top}\right)\right)$ and $h$ is a $\mathrm{T}^{\mathrm{T}}$-semi-homomorphism.

\section{REFERENCES}

[1] J. C. Abbott, "Semi-boolean algebra," Mat. Vestnik, no. 4, pp. 177-19, 1967.

[2] J. Araújo and M. Kinyon, "Independent axiom systems for nearlattice," Czechoslovak Mathematical Journal, no. 61, pp. 975-992, 2011.

[3] S. Celani and I. Calomino, "Stone style duality for distributive nearlattices," Algebra Universalis, vol. 71 , no. $127-153,2014$

[4] I. Chajda, R. Halaš, and J. Kühr, Semilattice Structures. Heldermann Verlag, Research and Exposition in Mathematics, 2007.

[5] I. Chajda, R. Halaš, and J. Zedník, "Filters and annihilators in implication algebras," Acta Univ. Palacki. Olomuc., Fac. rer. nat., Math., no. 37, pp. 41-45, 1988.

[6] I. Chajda and M. Kolařík, "Ideals, congruences and annihilators on nearlattices," Acta Univ. Palacki. Olomuc., Fac. rer. nat., Math. 46 (2007), ., no. 46, pp. 25-33, 2007.

[7] I. Chajda and M. Kolařík, "Nearlattices," Discrete Math., pp. 4906-4913., 2008.

[8] W. H. Cornish, "Normal lattices," Australian Mathematical Society. Journal. Series A. Pure Mathemathics and Statistic, vol. 14, pp. 200-215, 1972.

[9] W. H. Cornish and R. C. Hickman, "Weakly distributive semilattices," Acta Math. Acad. Sci. Hungar., vol. 32, pp. 5-16, 1978.

[10] R. Halaš, "Subdirectly irreducible distributive nearlattices," Miskolc Mathematical Notes, pp. 141$146,2006$.

[11] R. C. Hickman, "Join algebras," Communications in Algebra, vol. 8, pp. 1653-1685, 1980.

[12] M. Mandelker, "Relative annihilators in lattices," Duke Math. J., vol. 37, pp. 377-386, 1970.

[13] Y. S. Pawar and A. D. Lokhande, "Normal semilattices," Indian J. pure appl. Math., vol. 29, pp. 1245-1249, 1998.

[14] J. C. Varlet, "Relative annihilators in semilattices," Bull. Austral. Math. Soc., vol. 9, pp. 169-185, 1973.

Authors' addresses

Ismael Calomino

CONICET and Departamento de Matemáticas, Facultad de Ciencias Exactas, Univ. Nac. del Centro, Pinto 399, 7000 Tandil, Argentina

E-mail address: calominodexa.unicen.edu.ar

\section{Sergio Celani}

CONICET and Departamento de Matemáticas, Facultad de Ciencias Exactas, Univ. Nac. del Centro, Pinto 399, 7000 Tandil, Argentina

E-mail address: scelani eexa.unicen.edu.ar 